\title{
A STUDY FOR DETECTION OF MISSED TUBERCULOSIS CASES AT A TERTIARY CARE CENTRE IN NORTHERN BIHAR, INDIA
}

\author{
Kumar Saurabh', Yashwant Kumar Singh ${ }^{2}$, Ram Shanker Prasad ${ }^{3}$
}

${ }_{1}^{1}$ Senior Resident, Department of Microbiology, All India Institute of Medical Sciences, Jodhpur, Rajasthan.

2 Professor and Head, Department of Microbiology, Darbhanga Medical College, Laheriasarai, Bihar.

${ }^{3}$ Associate Professor, Department of Microbiology, Darbhanga Medical College, Laheriasarai, Bihar.

\begin{abstract}
BACKGROUND
ABSTRACT

In resource limited settings, the diagnosis of pulmonary tuberculosis heavily rests upon the sputum smear examination by ZN staining or Auramine-O staining. Despite all expertise, lots of cases are being missed at the DOTS and Designated Microscopy Centres (DMCs) under RNTCP. So, there is need to adopt measures to increase the detection rate of sputum smear microscopy.

The sputum samples declared negative for Acid fast bacilli by fluorescent staining method were decontaminated and concentrated by modified Petroff's method before performing ZN staining and were cultured to detect the missed positive cases.
\end{abstract}

\section{MATERIALS AND METHODS}

About 200 sputum samples which were declared negative after Auramine-0 staining at DOTS centre were selected and processed by modified Petroff's method before performing ZN staining. Furthermore, they were cultured by manual BBL MGIT technique and the positive tubes were confirmed for presence of Mycobacteria by ZN staining.

\section{RESULTS}

Out of 200 sputum samples, 9 samples (4.5\%) turned out to be positive by ZN staining after decontamination and concentration. Following MGIT culture 14 samples (7\%) got contaminated, while in 186 samples which could be followed for 6 weeks 49 samples (26.34\%) were found to be positive. The time for detection of Mycobacteria by MGIT ranged from about 5 to 13 days.

\section{CONCLUSION}

The study concludes that if decontamination and concentration technique like modified Petroff's method is adopted at DOTS and DMCs under RNTCP, the rate of detection of Mycobacteria will increase. Also, liquid culture methods like MGIT can be used for further enhancing the detection rate.

\section{KEYWORDS}

Tuberculosis, Mycobacterium Growth Indicator Tube (MGIT), Modified Petroff's Method, Ziehl-Neelsen Staining, Fluorescent Staining.

HOW TO CITE THIS ARTICLE: Saurabh K, Singh YK, Prasad RS. A study for detection of missed tuberculosis cases at a tertiary care centre in northern Bihar, India. J. Evolution Med. Dent. Sci. 2017;6(95):6971-6974, DOI: 10.14260/jemds/2017/1513

\section{BACKGROUND}

Tuberculosis (TB) remains to be the leading cause of morbidity and mortality in India. One-fourth of the global incidence of TB cases occur in India annually. As per WHO Global TB report, 2015 , out of the estimated global annual incidence of 9.6 million TB cases 2.2 million were estimated to have occurred in India. ${ }^{1}$ The Revised National Tuberculosis Control Programme (RNTCP), which is based on the Internationally recommended Directly Observed Treatment Short-Course (DOTS) Strategy since its launch in 1997 has expanded across the country in a phased manner. The twin objectives of the program were to achieve and maintain a cure rate of at least 85\% among new sputum positive (NSP) patients, and to achieve and maintain case detection of at least $70 \%$ of the estimated NSP cases in the community. ${ }^{2}$

'Financial or Other Competing Interest': None.

Submission 08-11-2017, Peer Review 05-12-2017,

Acceptance 11-12-2017, Published 23-12-2017.

Corresponding Author:

Dr. Kumar Saurabh,

Room No. 304, Resident Hostel,

AIIMS Residential Complex, Basni Phase-2,

Jodhpur,

Rajasthan-342005

E-mail:kr.saurabh14@gmail.com

DOI: $10.14260 /$ jemds $/ 2017 / 1513$

\section{(c) $($ ) $९$}

The nationwide network of designated sputum smear microscopy laboratories under RNTCP provides appropriate and accessible quality assured services for TB diagnosis. ${ }^{1}$ Direct sputum microscopy by Ziehl-Neelsen technique still remains the most important tool for detecting Mycobacterium tuberculosis after smear preparation from the yellow and most purulent part of the sputum. ${ }^{3}$ The importance of centrifugation and concentration methods (like modified Petroff's method) have been proved to increase the sensitivity of sputum smear examination for Mycobacterium tuberculosis by many studies, ${ }^{4}$ but still is not being followed at DOTS and Designated Microscopy Centres (DMCs) under RNTCP.

Fluorescent microscopy is potentially more sensitive and less labour intensive than traditional light microscopy using the Ziehl-Neelsen or Kinyoun method for the identification of acid-fast bacilli. Fluorescent microscopy is preferred in settings with large numbers of specimens submitted for suspected mycobacterial disease. ${ }^{5}$ If a TB suspect is found to be sputum smear positive, he receives proper treatment which is likely to cure him. But missed cases by virtue of sputum smear negative results can pose a serious threat to healthy community by spreading TB through droplet infection. Diagnosis in such cases can be done using advanced molecular techniques like nucleic acid amplification tests, ${ }^{6}$ but in resource limited settings the diagnosis still relies upon the $\mathrm{x}$ ray findings. 
Such diagnosis is very uncertain as no shadow is characteristic of TB and there is a high inter- and intra-reader variation. Also, $40 \%$ patients diagnosed as having TB by $\mathrm{x}$-ray alone may not have active TB disease. Culture still remains the Gold standard for diagnosis of Mycobacterium tuberculosis. Culture on solid media like LJ media takes around 8 to 12 weeks, whereas culture on liquid media like Middlebrook 7H9 take only around 6 weeks. Manual BBL MGIT system using Middlebrook 7H9 has a useful role in the routine diagnosis of pulmonary $\mathrm{TB}$, having a distinct advantage over the conventional LJ culture with respect to faster growth and better growth of fastidious mycobacterial species.7,8,9 This study was aimed at detection of Mycobacterium tuberculosis bacilli in those samples, which were declared negative after direct fluorescent microscopy.

\section{MATERIALS AND METHODS}

This was a prospective study carried out in the Department of Microbiology, Darbhanga Medical College and Hospital, Laheriasarai, from a period between March 2013 and June 2013.

\section{Source of Clinical Sample}

Patients attending DOTS Centre of Darbhanga Medical College and Hospital, Laheriasarai, for sputum smear examination formed the source of samples. As samples were already submitted to the DOTS Centre for AFB screening and no extra samples were collected from the patients, individual consent from the patients was not obtained. The age and sex of the patients were noted along with the history of anti-tubercular treatment if any. The sputum samples analysed in this study were from both new patients suspected of tuberculosis as well as from patients coming for followup.

\section{Inclusion Criteria for the Samples}

1. Sputum of patients, which were smear negative by direct fluorescent microscopy after Auramine- 0 staining at DOTS Centre, DMCH.

2. Quantity of sputum around $4-5 \mathrm{~mL}$ or more.

3. Freshly passed sputum samples or properly stored (refrigerated at $2-4^{0} \mathrm{C}$ ) samples, if delay in processing was anticipated.

\section{Exclusion Criteria for the Samples}

1. Blood mixed sputum sample, as N-acetyl-L-cysteine becomes ineffective in the presence of blood.

2. Pulmonary samples consisting mainly of saliva, clinical material on swabs or samples preserved in formalin.

\section{Specimen Collection}

Patients were instructed to collect at least $4-5 \mathrm{~mL}$ of an early morning sputum sample in wide mouthed, screw capped, sterile, leak-proof, disposable container at the DOTS centre. Smears were prepared for Auramine-O staining at RNTCP centre and fluorescent microscopy was done. A total of 200 such negative samples were randomly selected, one sample from each patient and were transferred to the Microbiology Laboratory. Samples were properly stored (refrigerated at $2^{0}$ $4^{0} \mathrm{C}$ ) if delay in processing was anticipated.

At the Microbiology Lab, initially smears were prepared for ZN staining before processing the samples. Specimens were then decontaminated and digested with $2 \% \mathrm{NaOH}$ and $\mathrm{N}$ -
acetyl-L-cysteine for 15 mins. The reaction was stopped by addition of phosphate buffer followed by centrifugation at $2500 \mathrm{x}$ g for 15 mins (modified Petroff's method). ${ }^{4}$ Smears were prepared from the deposit. ZN staining was performed on these smears and microscopy was done to search for the presence of Mycobacterium tuberculosis if any. Furthermore, all the samples were cultured in Middlebrook 7H9 medium using Manual BBL MGIT system as per the protocols 10 and were followed upto 6 weeks for Mycobacterial growth. Positive and negative control tubes were put up with each batch of culture. Readings were taken using the manual MGIT reader daily for first week and thereafter twice weekly upto 6 weeks. If the readings were positive, the growth was confirmed by $\mathrm{ZN}$ staining. If the reading was positive and the $\mathrm{ZN}$ staining was negative, some of the materials from the tube was inoculated on Blood agar. The inoculated blood agar plate was incubated for $48 \mathrm{hrs}$. If there was any growth on blood agar gram staining was done and if any microorganism was visible the tube was labelled as contaminated. These contaminated tubes were excluded from the study. If there was no growth on Blood agar plate, the tubes were labelled as uncontaminated and were followed for 6 weeks with a weekly ZN smear. At the end of 6 weeks, if ZN staining from a previously positive tube (by MGIT reader) was negative it was finally labelled as negative. All samples were processed in a Class-2 Bio-safety Cabinet and all precautions were undertaken to avoid any possible smear contamination.

\section{Statistical Analysis}

The collected data were entered in MS Excel Sheet and analysed using SPSS version 17. Results were expressed in percentages and displayed in tabular form.

\section{RESULTS}

Out of total 200 sputum samples, 141 (70.5\%) samples were taken from male and 59 (29.5\%) samples were taken from female patients. After the decontamination and concentration process, 9 samples (4.5\%) turned out to be positive for Mycobacteria by ZN staining. Furthermore, when the samples were inoculated in MGIT and incubated, 14 (7\%) of them got contaminated and were excluded from the study. Out of 186 MGIT tubes, which could be followed till 6 weeks 49 tubes (26.34\%) showed positive growth for Mycobacteria (including the 9 samples which were positive after decontamination and concentration process), which was further confirmed by ZN staining. AFB appeared red in colour in blue background, growing singly and in cords (Figure 1). Age wise distribution of 186 samples and the final result is shown in Table-1.

A maximum of $50 \%$ detection was done in the age group of 61 - 80 years, whereas out of 2 samples from patients of age group 81 - 100 one was found to be positive. Minimum positivity (14.28\%) was in the age group of $1-20$ years. Time to detection of growth in MGIT was noted in each case. The average number of days to positivity was 12.62 in those cases, which remained negative by ZN staining after the initial decontamination and concentration process, but it was only 5 . 22 days in those 9 cases, which were found to be positive (Table-2). Treatment history was also noted in all the positive cases which showed that out of 49 positive cases 18 were on Category I treatment, 16 were on Category II treatment, whereas 15 of them were not taking any treatment (Table-3). 


\begin{tabular}{|c|c|c|c|}
\hline $\begin{array}{c}\text { Age } \\
\text { Groups } \\
\text { (Years) }\end{array}$ & $\begin{array}{c}\text { Positive MGIT } \\
\text { Results } \\
\text { (AFB Positive) }\end{array}$ & $\begin{array}{c}\text { Negative MGIT } \\
\text { Results } \\
\text { (AFB Negative) }\end{array}$ & $\begin{array}{c}\text { Total } \\
\text { (n= 186) }\end{array}$ \\
\hline $1-20$ & $03(14.28)$ & $18(85.71 \%)$ & $21(11.29 \%)$ \\
\hline $21-40$ & $19(22.09 \%)$ & $67(77.91 \%)$ & $86(46.24 \%)$ \\
\hline $41-60$ & $16(28.07 \%)$ & $41(71.92 \%)$ & $57(30.64 \%)$ \\
\hline $61-80$ & $10(50.00 \%)$ & $10(50.00 \%)$ & $20(10.75 \%)$ \\
\hline $81-100$ & $01(50.00 \%)$ & $01(50.00 \%)$ & $02(1.07 \%)$ \\
\hline Total & 49 (26. 34\%) & 137 (73. 65\%) & 186 (100\%) \\
\hline Table 1. Age Wise Distribution of Sputum Samples and the \\
Final Result after MGIT Culture (n= 186) \\
\hline
\end{tabular}

\begin{tabular}{|c|c|c|}
\hline $\begin{array}{c}\text { Status of Samples } \\
\text { after Processing }\end{array}$ & $\begin{array}{c}\text { No. of Positive } \\
\text { Samples by MGIT }\end{array}$ & $\begin{array}{c}\text { Time to } \\
\text { Detection (Days) }\end{array}$ \\
\hline AFB positive & 09 & 05.22 \\
\hline AFB negative & 40 & 12.62 \\
\hline Table 2. Time to Detection of Growth by Manual MGIT \\
Culture Method \\
\hline
\end{tabular}

\begin{tabular}{|c|c|}
\hline $\begin{array}{c}\text { Treatment being administered } \\
\text { to the Patients }\end{array}$ & $\begin{array}{c}\text { No. of Patients whose Samples } \\
\text { were Positive by MGIT Culture }\end{array}$ \\
\hline Category 1 regimen & 18 \\
\hline Category 2 regimen & 16 \\
\hline No treatment & 15 \\
\hline Total & 49 \\
\hline $\begin{array}{c}\text { Table 3. Anti-Tubercular Treatment (ATT) being } \\
\text { administered to the Positive Patients }\end{array}$ \\
\hline
\end{tabular}

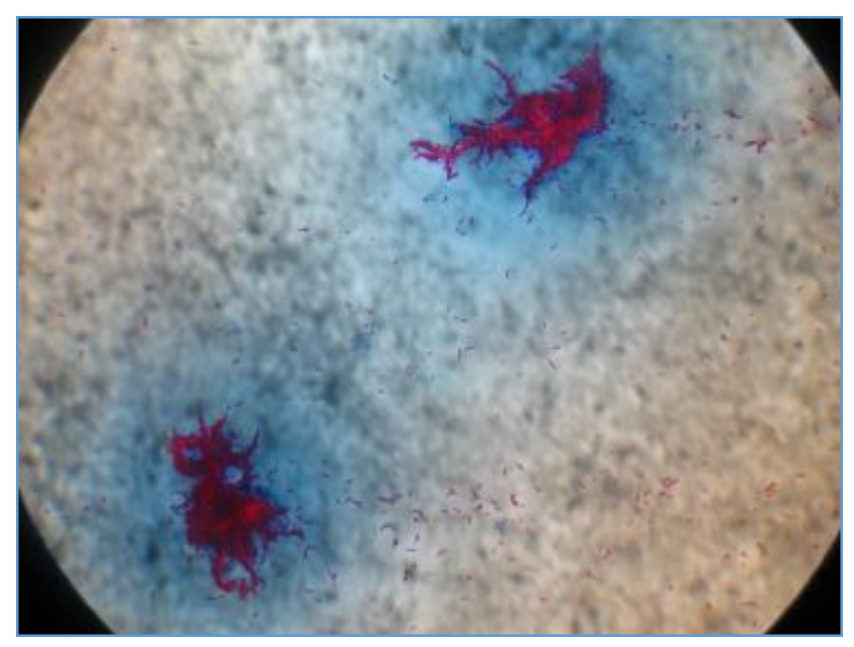

Figure 1. Mycobacterium tuberculosis appearing Singly and in Cords

\section{DISCUSSION}

It would not be an extrapolation to state that in resource limited settings often the diagnosis of tuberculosis is missed in sputum smear negative cases. DOTS centre have started to function at many places under RNTCP, but yet TB control seems to be a nightmare. Out of 200 negative samples, 9 samples $(4.5 \%)$ turned out to be positive by ZN staining after adopting the decontamination and concentration technique (modified Petroff's method). This indicates that routine concentration of sputum before making smears can really enhance the rates of detection of AFB. Chintapalli $S$ et al detected $11(9.1 \%)$ additional positive samples out of 120 negative samples, after modified Petroff's method. ${ }^{11}$ The lower percentage of detection in our study may be due to the fact that the included samples were initially declared negative after direct fluorescent staining, which is a more sensitive method as compared to direct $\mathrm{ZN}$ staining which was used by
Chintapalli S et al. Another study showed the increase in sensitivity of ZN staining by $6.67 \%$ after concentration method.12 Peterson et al also concluded in his study that the direct smear was always less sensitive than the smear made from the concentrated specimens. ${ }^{4}$

Fluorescent staining has been proved to be a more sensitive method as compared to ZN staining12,13,14,15,16,17,18 and is being used at many DOTS centres and DMCs nowadays. But this study shows that many cases are being missed even after using fluorescent staining and that ZN staining after Petroff's method is superior to direct fluorescent staining, as out of total 200 negative samples (after direct fluorescent staining) 9 samples (4.5\%) were found to be positive by ZN staining after concentration. Also, out of 186 sputum samples which could be followed till the end of 6 weeks, 49 samples (26.34\%) turned out to be positive for Mycobacteria by MGIT culture method. If we compare the results of $\mathrm{ZN}$ staining after concentration and manual MGIT culture, only $4.5 \%$ cases could be diagnosed by the former, whereas $26.34 \%$ cases were found to be positive by the later. Similar rate of detection (around 24\%) in negative sputum samples was reported by $\mathrm{T}$ Battaglioli et al by manual MGIT culture technique. ${ }^{7}$ Another study using manual BBL MGIT showed 19.8\% positivity. ${ }^{9}$ Slightly higher detection rate $(39.18 \%)$ was reported by using automated MGIT 960 method out of 222 negative pulmonary samples by $\mathrm{S}$ Rishi et al. ${ }^{19}$ This higher detection rate may be attributed to the automated MGIT 960 method being used in comparison to the manual MGIT method, which was used in our study. The average time to detection in smear positive samples was 5.22 days and in smear negative samples was 12.62 days, which is in accordance with the range of days reported by other studies.7,9,19,20,21 14 samples out of 200 samples (7\%) got contaminated by using BBL MGIT technique, which could not be followed till 42 days. This rate of contamination $(7 \%-8 \%)$ is acceptable while using liquid culture media. Very low contamination rate $(<3 \%)$ may indicate too harsh a decontamination process, which would also affect growth of mycobacteria and may reduce the positivity rate and increase time to detection of positive mycobacterial culture. Contamination rate above $8 \%$ may be due to improper or under decontamination, very mucoid specimens, long storage and transportation time of specimen after collection or use of non-sterile materials as pipettes, tubes etc. 10

Lastly, out of the 49 positive cases, 18 were on Category I ATT and 16 cases were on Category II ATT, which implicates that they might be cases of Multidrug resistant (MDR) TB. ${ }^{22}$ Furthermore, 15 cases were not taking any treatment and neither were they prescribed any ATT, as they could not be diagnosed at the DOTS centre. Altogether, these cases which were missed can prove to be a potential source of infection to the healthy individuals and may cause spread of MDR TB.

\section{Limitations}

Biochemical tests could not be performed on the positive samples to differentiate between the members of MTBC and NTM, which could have additionally helped in the estimation of missed cases of NTM infections, which closely mimics tuberculosis. Solid culture on LJ media could not be performed simultaneously with MGIT culture as done in many studies, which could have added to the existing data proving the usefulness of MGIT in terms of sensitivity and rapid time to 
detection of Mycobacterium tuberculosis. Blood mixed sputum samples were excluded as $\mathrm{N}$-acetyl-L-cysteine, which is being used for mucolysis of sputum samples, becomes ineffective in the presence of blood. "In case of inclusion of these samples with treatment by Petroff's method (with sodium hydroxide as a mucolytic agent), there are chances to increase the numbers of AFB positive cases.

\section{CONCLUSION}

When the sputum samples turn out to be negative after $\mathrm{ZN}$ or fluorescent staining, the diagnosis of TB rests upon nonspecific tests like $x$-ray chest in resource limited settings. These patients are either injudiciously treated for TB or are denied the treatment in genuine cases. Even in followup cases, it becomes hard to judge whether the cases are truly responding or are they drug resistant cases (MDR-TB). Implication of a simple procedure like modified Petroff's method routinely to all sputum samples at DOTS centre and DMCs can definitely increase the detection of AFB after ZN staining. Liquid culture methods like MGIT can really help in detecting the positive TB cases in less than 2 weeks' time and starting ATT in these cases, thereby reducing the spread of TB.

\section{ACKNOWLEDGEMENTS}

We convey our humble thanks to all the staffs of Microbiology Department and specially Mr. Girija Shankar Jha from DOTS centre, Darbhanga Medical College, Laheriasarai.

\section{REFERENCES}

[1] TB INDIA 2016. Revised National TB Control Programme Annual status report PART-1. iapsm.org/pdf/reports/Annual Report TB India 2016.pdf

[2] Sachdeva KS, Kumar A, Dewan P, et al. New vision for Revised National Tuberculosis Control Programme (RNTCP): Universal access - reaching the un-reached. Indian J Med Res 2012;135(5):690-4.

[3] Revised National Tuberculosis Control Programme. DOTS-Plus Guidelines. Government of India. Central TB Division, Directorate General of Health Services, Ministry of Health and Family Welfare, Nirman Bhawan, New Delhi, 2010. health.bih.nic.in/Docs/Guidelines/Guidelines-DOTSPlus.pdf

[4] Peterson EM, Nakasone A, Platon-DeLeon JM, et al. Comparison of direct and concentrated acid-fast smears to identify specimens culture positive for Mycobacterium spp. J Clin Microbiol 1999;37(11):3564-8.

[5] Kuhn W, Armstrong D, Atteberry S, et al. Usefulness of the paralens fluorescent microscope adaptor for the identification of mycobacteria in both field and laboratory settings. Open Microbiol J 2010;4:30-3.

[6] Katoch VM. Newer diagnostic techniques for tuberculosis. Indian J Med Res 2004;120(4):418-28.

[7] Battaglioli T, Soto A, Agapito J, et al. Manual liquid culture on simple Middlebrook 7H9 or MGIT for the diagnosis of smear-negative pulmonary tuberculosis. Trop Med Int Health 2014;19(12):1500-3.

[8] Rawat J, Biswas D, Sindhwani G, et al. Diagnostic role of MGIT culture of BAL samples in sputum smear negative pulmonary tuberculosis. Indian J Tuberc 2013;60: 77-82.

[9] Fadzilah MN, Kee PNG, Yun FN. The manual MGIT system for the detection of $M$. tuberculosis in respiratory specimens: an experience in the University Malaya Medical Centre. Malays J Pathol 2009;31(2): 93-7.

[10] Siddiqi SH, Rusch-Gerdes S. MGIT Procedure manual (FIND) 2006. https://www.finddx.org/wpcontent/uploads/2016/02/mgit_manual_nov2006.pdf

[11] Chintapalli S, Krishna PB, Sivajyothi P, et al. Comparision between different conventional methods for the diagnosis of mycobacterium tuberculosis. J Evol Med Dent Sci 2015;4(38):6631-5.

[12] Hooja S, Pal N, Malhotra B, et al. Comparison of Ziehl Neelsen \& Auramine 0 staining methods on direct and concentrated smears in clinical specimens. Indian J Tuberc 2011;58(2):72-6.

[13] Githui W, Kitui F, Juma ES, et al. A comparative study on the reliability of the fluorescence microscopy and ZiehlNeelsen method in the diagnosis of pulmonary tuberculosis. East Afr Med J 1993;70(5):263-6.

[14] Ba F, Rieder HL. A comparison of fluorescence microscopy with the Ziehl-Neelsen technique in the examination of sputum for acid-fast bacilli. Int J Tuberc Lung Dis 1999;3(12):1101-5.

[15] Laifangbam S, Singh HL, Singh NB, et al. A comparative study of fluorescent microscopy with Ziehl-Neelsen staining and culture for the diagnosis of pulmonary tuberculosis. Kathmandu Univ Med J 2009;7(27):22630.

[16] Zaib-un-Nisa, Javed H, Zafar A, et al. Comparison of fluorescence microscopy and Ziehl-Neelsen technique in diagnosis of tuberculosis in paediatric patients. J Pak Med Assoc 2015;65(8):879-81.

[17] Kivihya-Ndugga LEA, Van Cleeff MRA, Githui WA, et al. A comprehensive comparison of Ziehi-Neelsen and fluorescence microscopy for the diagnosis of tuberculosis in a resource-poor urban setting. Int J Tuberc Lung Dis 2003;7(12):1163-71.

[18] Jagadeesha K, Pai V. A comparative study of Fluorescent staining and Ziehl Neelsen's staining for detection of acid fast bacilli in sputum in a tertiary care hospital in Mangalore. Int J Microbiol Parasitol 2015;1(1):1-3.

[19] Rishi S, Sinha P, Malhotra B, et al. A comparative study for the detection of mycobacteria by BACTEC MGIT 960, Lowenstein Jensen media and direct AFB smear examination. Indian J Med Microbiol 200;25(4):383-6.

[20] Mishra V, Sami H, Bareja R, et al. Evaluation of MGIT over other phenotypic methods for the detection of pulmonary and extrapulmonary TB at a tertiary care centre in North India. Int J Pharm Sci Res 2016;7(6):2568-72.

[21] Pfyffer GE, Welscher HM, Kissling P, et al. Comparison of the mycobacteria growth indicator tube (MGIT) with radiometric and solid culture for recovery of acid-fast bacilli. J Clin Microbiol 1997;35(2):364-8.

[22] Catanzaro A, Rodwell TC, Catanzaro DG, et al. Performance comparison of three rapid tests for the diagnosis of drug-resistant tuberculosis. PLoS One 2015;10(8):e0136861. 